\title{
LIII. On the mechanical theory of Crookes's (or polarization) stress in gases
}

\section{G. Johnstone Stoney M.A. F.R.S.}

To cite this article: G. Johnstone Stoney M.A. F.R.S. (1878) LIII. On the mechanical theory of Crookes's (or polarization) stress in gases, Philosophical Magazine Series 5, 6:39, 401-423, DOI: $10.1080 / 14786447808639535$

To link to this article: http://dx.doi.org/10.1080/14786447808639535

曲 Published online: 13 May 2009.

Submit your article to this journal $[\pi$

Џ Article views: 2

Q View related articles $\square$ 
LONDON, EDINBURGH, AND DUBLIN

\title{
PHILOSOPHICAI MAGAZINE
}

\author{
AND \\ JOURNAL OF SCIENCE.
}

[FIFTH SERIES.]

DECEMBER 1878.

LIII. On the Mechanical Theory of Crookes's (or Polarization) Stress in Gases. By G. JoHnstone StoneY, M.A., F.R.S., \&c., Secretary to the Royal Dublin Society*.

\section{Introduction.}

TWO papers will be found in the first volume of the fifth 1 series of the Philosophical Magazine (March and April 1876), in which I endeavoured to explain the force that Mr. Crookes bad detected within vacuum-chambers, by pointing out that when heat passes across the residual gas, the molecules of the gas that tend respectively towards the heater and towards the cooler must interpenetrate one another in a greater degree than they would if the gas were in its ordinary or unpolarized condition, and that this behaviour will render the stresses within the gas unequal, causing the stress to be greatest in the direction in which the augmented interpenetration takes place.

When writing the foregoing papers, and afterwards when writing a paper on the transfer of heat which accompanies the phenomenon, I was under the mistaken impression that the flow of heat between a heater and cooler in fixed positions, and at constant temperatures, will become greater if the number of gaseous molecules that intervene is reduced below the number required for the transfer of heat by the laws of "con-

* From the 'Scientific Transactions of the Royal Dublin Society' for the year 1878. Read February 18, 1878. Communicated by the Author. Phil. Mag. S. 5. Vol. 6. No. 39. Dec. 1878. 
duction"*; and for this supposed increased flow of heat I suggested the name penetration. It has recently been pointed out by Dr. Schuster ('Nature,' vol. xvii. p. 143) that experiments have been made which show that the flow of heat diminishes instead of increasing when the limit for "conduction" is passed. It thus appears that what I have called penetration is always feebler than conduction, and is to be sought, in the figures representing De la Provostaye and Desains's experiments, in those portions of the curves which slope steeply downwards. Accordingly my paper on Penetration (Phil. Mag. December 1877), and especially that part of it in which I apply the theory to experiment, requires considerable modification, and some of the statements I made in my earlier papers on Crookes's force need amendment. Although the corrections that are required do not affect any material part of the theory of unequal stresses within polarized gas, it has appeared desirable to resume the subject and present the theory freed from the error that has been pointed out. In doing this I have taken the opportunity of introducing the conception of the reffecting tube, which greatly facilitates the inquiry into the mechanical effect of the interpenetration; and I have also availed myself of the admirable method of treating the problem, described by Mr. George F. Fitzgerald in 'Nature,' vol. xvii. p. 200, to obtain a complete expression for the stress, and to show that my theory is not at variance with results established by Professor Clausius, as has been asserted by Professor Osborne Reynolds in 'Nature,' vol. xvii. p. 122 .

\section{Part I.-Treatment of the Problem by General Mechanical Considerations.}

1. If a drop of water or other volatile liquid is allowed to fall into a smooth and sufficiently hot metal dish, it continues a liquid drop instead of spreading out or flashing off into vapour, and it exhibits an appearance of great mobility. The drop is then in what has been called the "spheroidal state." Now, when a drop of liquid is so situated a chink may be observed between it and the hot surface beneath; so that the drop does not rest directly upon the metal, but is in reality floating upon a layer of vapour. We further learn

* It is known that gases feebly conduct heat by diffusion, and that the amount of heat which passes in this way between a heater and cooler is independent of the density of the intervening gas, provided that the density of the gas does not fall below a certain limit. The question that presented itself was as to, what happens below that limit. 
from these observations that after the brief interval of adjustment is over the layer of vapour presses upwards and downwards more than it presses sideways ; for the pressure sideways must equal the pressure of the atmosphere so soon as the adjustment is over, otherwise air would still be entering or leaving the chink, whereas the pressure upwards must exceed the pressure of the atmosphere by an amount able to support the drop. It is my object to explain how this difference of pressures, this Crookes's pressure as it has been called, comes into existence.

2. The thermal conditions of the problem are easily traced, but need not detain us here. It is enough to state that they show the metal dish and drop to be at different temperatures, so that they are a heater and cooler on either side of the layer of vapour. Experiment further shows that the heater and cooler may be either one a liquid and one a solid, as in the case already considered, or both liquids, or both solid, and that the intervening layer may be either vapour or permanent gas. This last important fact has been established by Mr. Richard Moss in an admirable series of experiments lately made by him to test the theory of the present communication (see 'Scientific Proceedings of the Royal Dublin Society,' vol. i. p. 89). It is also found to be immaterial whether the heater or cooler is uppermost, or whether they face one another sideways.

Other facts of importance have been elicited by the experiments at low tensions, of which the most significant are:- that when the heater and cooler are maintained at given temperatures the Crookes's stress between them may be increased either by bringing the heater and cooler closer together, or by attenuating the gas until a certain point is reached which varies from one gas to another; and that when that point is passed, the force decrenses and apparently without limit.

3. We may express these facts in a very convenient form for our present purpose if the heater and cooler are extensive flat parallel surfaces at fixed temperatures. Conceive two exactly similar patches on the heater and cooler directly opposite to each other, and each occupying a unit of surface, and consider that portion of space which lies between these. Then the observations show that there is one definite quantity of the gas to be left in the volume so marked out, if we wish to produce the strongest Crookes's stress. And, further, by comparing Mr. Crookes's experiments on the mechanical action with those of De la Provostaye and Desains on the flow of heat, we learn another important fact, viz. that the maximum stress occurs when the quantity of gas is too little to admit of $2 \mathrm{D} 2$ 


\section{Mr. G. J. Stoney on Polarization Stress in Gases.}

the passage of heat under the laws of the conduction of heat in gases. Now these facts also follow as consequences from the theory advanced by the author, and therefore become confirmations of it.

4. This theory seeks to account for Crookes's force by showing that a layer of gas placed between a heater and cooler is in a polarized condition of such a kind that the stresses within the gas are different in different directions. Gas is polarized whenever the molecules within a spherical element of volume are moving towards different quarters with numbers or velocities that are not distributed alike in all directions, the velocities being measured from the centre of mass of these molecules. This definition excludes the case of mere wind, which is to be regarded as unpolarized gas travelling forward in a certain direction; but it includes the case of gas across which heat is making its way, which is the case with which we have here to deal.

5. Let us recur to the simple instance of a heater and cooler with extensive flat parallel surfaces maintained at constant temperatures, and with gas between them freed from the action of gravity, and which has had time to adjust itself to its position. Gas so circumstanced will become stationary in the ordinary sense of the word; i.e., though in active molecular motion, it will have no currents like convectioncurrents or wind passing through it *. We have now to show that the stress across such a layer will be greater than the stress sideways.

6. Imagine a unit of suface marked out on either heater or cooler, and let perpendiculars to the surface be raised from the boundary of this enclosure. These will trace out a straight tube extending between the heater and cooler, and closed at the ends by equal patches of the heater and cooler. These we may call the pistons of the tube. The molecules which strike the pistons are returned by them, and with altered velocity whenever the pistons are at different temperatures ; but molecules pass without hindrance through the sides of the tube. Now it is evident that, if the molecules passing through an element of the side of the tube are considered, those passing out in a unit of time will be an exact counterpart of those passing in, in such a sense that the state of the gas would not be disturbed by making the sides of the tube im-

* There will be currents close to the boundary of the heater and cooler; but these are secondary phenomena caused by, and in no degree the cause of, Crookes's stress. They will not be appreciable within the layer at any considerable distance from the edge; and they may be avoided by giving to the opposed surfaces of the heater and cooler the form of concentric spheres. 
pervious to molecules, provided that they were made at the same time perfect reflector's of molecules. By a reflector of molecules is to be understood a surface endowed with the property of throwing off any molecules that impinge upon it with unabated speed, and at an angle of reflection which lies in the same plane as the angle of incidence, and is equal to it. The reflected molecules will affect the state of the gas within the tube exactly in the same way as the molecules passing in from outside had done before. W $W_{e}$ have now a portion o, gas completely shut up inside a tube with sides that are perfect reflectors of molecules, and closed at the ends by pistons that are patches of the heater and cooler, and which therefore scatter such molecules as reach them; and we know that the behaviour of this gas will be the same as that of the corresponding portion of the Crookes's layer. We may call such a tube a unit reflecting tube.

7. Let the pistons of such a tube be kept at the temperatures $\mathrm{T}_{1}$ and $\mathrm{T}_{2}$, and let gas be introduced into it. After a brief period of adjustment the gas will become stationary; $i$.e., if a plane forming a cross section of the tube be considered, the molecular motions are such that the same number of molecules pass forwards as backwards through this plane per second. But they will pass it with unequal average velocities, because the ris viva of those crossing it towards the cooler must exceed the vis viva of those crossing it towards the heater by an amount bearing a known ratio to the quantity of heat advancing. Hence the gas is polarized, the molecular motions being swifter when they are directed forward or towards the cooler, and slower when directed backwards.

8. Suppose that we begin with dense gas and gradually exhaust, and let us consider the succession of events that will arise as the exhaustion proceeds, i. e. when $n$, the number of molecules in the unit tube, is progressively diminished. It is known that the flow of heat cannot conform to the laws of "conduction" unless the number of molecules exceeds a certain limit which we may call $\mathrm{N},--\mathrm{N}$ depending upon the description of gas that is present, and upon the temperatures $T_{1}$ and $\mathrm{T}_{2}$ of the pistons which close the unit tube. We must therefore divide the exhanstion into two periods, one lasting while the number of molecules in the tube exceeds $\mathrm{N}$, and the other during the rest of the exhaustion. Throughout the first period the flow of heat follows the known laws of conduction, and therefore remains constant. Hence, during this part of the exhaustion the polarization of the gas (which may be measured by $\frac{\delta v}{v}, v$ being the average velocity at any point 
106 Mr. G. J. Stoney on Pularization Stress in Gases.

of the layer, and $\delta v$ the average difference of the velocities forwards and backwards at that station) is so rapidly on the increase as quite to compensate in $K \rho v^{2} \delta v$ (the expression for the flow of heat, $\rho$ being the density at the station, and $\mathrm{K}$ a constant) for the diminishing density. During the second period, i. e. when the molecules have become fewer than $\mathrm{N}$, the polarization is still on the increase, but not so rapidly as before, and at the same time the flow of heat decreases to zero; for while $\rho$ tends to zero as the exhaustion proceeds, the polarization does not tend to infinity, but to a limit, viz. $\frac{v_{1}-v_{2}}{\sqrt{v_{1} v_{2}}}$, where $v_{1}$ and $v_{2}$ are the velocities corresponding to $\mathrm{T}_{1}$ and $\mathrm{T}_{2}$, the temperatures of the pistons. Now, when gas is polarized with this kind of polarization within a tube the sides of which reflect the molecules, we can find limits between which its thermal and mechanical properties must lie.

9. Before proceeding to determine these limits, it will be well to guard ourselves against making mistakes by passing under review the orders of the several magnitudes with which we are dealing in this inquiry. No accurate measures appear yet to have been made of the thickness of the chinks of air or vapour on which spheroidal drops rest. But from approximate measures, some of which were made by Mr. Fitzgerald and some by myself, I think it may be inferred that this thickness is somewhere about the thickness of a sheet of paper (i.e. about a fourth-metre or the tenth of a millimetre) when a spheroidal drop of the density of water, at a temperature of $10^{\circ} \mathrm{C}$. and 5 or 6 millims. in diameter, floats over a surface of liquid which is about $10^{\circ}$ warmer. We further know that in this case the Crookes's pressure, as it supports the weight of this drop, must be about the twothousandth part of an atmosphere. These determinations are very rude; but they at all events tell us what kind of magnitude we are dealing with, and therefore suffice for our present purpose. They show that we shall not be far wrong in assuming definitively that the phenomenon presented by experiment which we have to explain is, that the stress across a stratum of air will be $\frac{1}{2000}$ part of the stress at right angles to that direction, if this stratum occupies the space between a heater and cooler at temperatures of $10^{\circ}$ and $20^{\circ} \mathrm{C}$., if, moreover, this interval is a fourth-metre (a metre divided by $10^{4}$ ), and if the atmosphere has free access to the stratum of air at its edges. Let us now imagine a reflecting tube, such as is described above, to be placed across this stratum. It will therefore be a fourth-metre long ; and we may assign to.it 
any width we please. Let us take a width equal to the diameter of the smallest object that can be seen with a microscope, which is about $2 \cdot 5$ seventh-metres, or the 100,000 th part of an inch. Wo have now to compare the dimensions of this tube with the number and motions of the molecules included within it. The number of molecules in a cubic millimetre of atmospheric air is about a unit-eighteen $\left(10^{18}\right)$. (See Phil. Mag. August 1868.) Whence the average interval between them is about a ninth-metre. This is the 100,000th part of the length of our tiny tube and the 250th part of its breadth. Hence the tube will contain a vast number of molecules, some such number as five thousand millions. Again, the average striking-distance (i. $e$. the average length of path between the encounters) of the molecules is about the 1500 th part of the length of the tube, or the fourth part of its breadth. There is, therefore, abundant room within the tube, small as it is, for a vast number of molecules and for much jostling amongst them. The temperatures with which we are dealing are such that the average velocity with which the molecules of air dash about may be taken as 500 metres per second; and the molecules meet with so many encounters, that the direction of the path of each is changed somewhere about a unit-ten of times $(10,000,000,000)$ every second. To complete the picture, we must remember that each molecule is in a state of vigorous internal motion as well as travelling about among its fellows, and that when an encounter takes place, the energy which passes from one molecule to another is employed in changing both those kinds of motion, and possibly (but not probably) another part becomes potential energy, $i$. e. energy expended in altering the configuration of the parts of the molecule, or the position of its parts with reference to the rether. The motions which go on within the molecules are what give rise to the linear spectra of gases, and are therefore those motions of the gas that act on the æother, and are in turn partly controlled by it*. They are recurring motions which, at least in some cases, are resolvable either

* May we not look, with some prospect of success, to the control which is exercised by the ether on the internal motions of the molecule for the explanation of the number of " degrees of freedom" of a molecule, which (on the supposition that there is no potential energy) is in most gases 5 (see Watson's 'Kinetic Theory of Gases,' p. 39). The number 5 appears to indicate that the motions within the molecules are trammelled, as here suggested. This view is, moreover, supported by the fact that light is emitted by the gas, which could not be the case unless vast numbers of molecules noved in unison with one another; and the most probable account of this appears to be that they are-all trammelled in the same way by their common relation to the ather. 


\section{Mr. G. J. Stoney on Polarization Stress in Gases.}

into harmonics like the vibrations of a string, or else into quasi-harmonics not to be distinguished from harmonics by observation (see Donkin's 'Acoustics,' 194), like the transverse vibrations of an elastic rod-probably the former. On the more probable supposition that they are true harmonics, the periodic times have been determined with great precision in some cases, notably in the cases of a motion within the molecules of hydrogen, which gives rise to three of its spectral lines, and a motion within the molecules of chlorochromic anhydride, which gives rise to 105 of its spectral lines. In hydrogen the motion is repeated as often as $2,280,000,000,000$ times each second in every molecule, and in the vapour of chlorochromic anbydride rather more than $800,000,000,000$ times*. Such are the periodic times on the supposition that the motions are resolvable into true harmonics; and whether the fact be that the components of the motions are harmonics or quasi-harmonics, their periodic tines are at all events quantities of this order. The general presumption, therefore, is that the periodic times within the molecules of other gases are also quantities of this order. But it is not necessary for our present purpose to establish this. The only circumstance relating to these inner motions with which we are here directly concerned is that the energy which is transferred from molecule to molecule is employed partly in altering the relocities with which the molecules travel about, and partly in altering these internal motions and (perhaps) collocations, and that the proportion of the energy which is employed in the former way bears on the average a numerical ratio to the whole energy transferred which can be determined experimentally (see Maxwell's ' Theory of Heat,' p. 299) and is denoted in the sequel by $\frac{1}{\beta}$.

10. We now proceed to determine limits between which the thermal and mechanical properties of the gas must lie. For this purpose let us imagine a tube of the kind described

* The periodic times deduced from the observations are respectively $\frac{\tau}{76 \cdot 18}$ and $\frac{\tau}{2 \cdot 70^{\prime}}, \tau$ being the time that ight takes to advance $1 \mathrm{millim}$. in vacuo. (See Phil. Mag. A pril 1871, p. 295, and July 1871, p. 45. In the former paper read 0.013127714 for 0.13127714.) The first of these determinations was made by the present author, and the second by the present author in conjunction with Professor Fmerson Reynolds, of Dublin: but, before either of these determinations were made, Professor Clifton, of Orford, had mentioned at the Exeter Meeting of the British Association in 1869 that he had found two of the hydrogen lines (probrbly $\mathrm{C}$ and $\mathrm{h}$ ) to be related harmonically. I am not aware that any record of this important observation has been published. 
above, with perfectly reflecting sides. Such a tube exerts no friction on gas flowing along it, nor does it occasion any loss of energy. Let it contain a large number of gaseous molecules between pistons at temperatures $T_{1}$ and $T_{2}$. And let us further suppose that the molecules of the gas, as they leave either piston, acquire the property of not interfering with or being obstructed by the molecules that have last left the other. This imaginary state of the gas would re sult in two streams constantly travelling in opposite directions along the tube. Iet us follow one of these streams. It starts from its piston with a mean of the squares of the velocities of its molecules $v_{1}^{2}$ determined by the temperature of the piston, and in numbers per unit of time represented by $\rho^{\prime} u^{\prime}$, $\rho^{\prime}$ being the density of the stream and $u^{\prime}$ the average of the normal components of the velocities at starting. Then, however the velocities and directions may have been distributed at starting, the jostling of the molecules of this stream among one another will reduce the stream as it advances to the condition of unpolarized gus travelling along the tube with the velocity $u^{\prime}$. The molecules are henceforward moving with velocities among themselves which, measured from their advancing centre of mass, have an average square of the velocities $w^{\prime 2}$ which is given by the equation

$$
\beta v_{1}^{2}=u^{\prime 2}+\beta w^{\prime 2}, \text {. . . . . . }
$$

$\beta$ being the known numerical coefficient representing the ratio of the total energy of the gas to its "energy of agitation." This equation is only the symbolical expression of the fact that no energy has entered or left the gas. The stream moving in the opposite direction furnishes the similar equation.

$$
\beta v_{2}^{2}=u^{\prime 2}+\beta w^{\prime \prime 2} \text {. . . . . . }
$$

And since the numbers of molecules reaching and receding from each piston are equal, we have the further equation

We have also

$$
\rho^{\prime} u^{\prime}=\rho^{\prime \prime} u^{\prime \prime} . \quad \text {. . . . . . . }
$$

$$
\rho=\rho^{\prime}+\rho^{\prime \prime} . \quad \cdot \quad \cdot \cdot \cdot \cdot \cdot \cdot
$$

Of the quantities which enter into these equations, $\rho$, the density of the gas, is known, and $v_{1}, v_{2}, u^{\prime}$, and $u^{\prime \prime}$ are known functions of $\mathrm{T}_{1}$ and $\mathrm{T}_{2}$, the temperatures of the pistons. Hence these equations enable us to determine the remaining quantities $\rho^{\prime}, \rho^{\prime \prime}, w^{\prime}$, and $u^{\prime \prime}$.

Now, under the conditions that have been laid down, it is 
manifest that the stress * of the gas sideways would be

$$
\mathrm{P}_{y}=\frac{1}{3} \rho^{\prime} w^{\prime 2}+\frac{1}{3} \rho^{\prime \prime} w^{\prime \prime 2} \text {, . . . . . . . }
$$

while the stress along the tube would be

$$
\mathrm{P}_{x}=\frac{1}{3} \rho^{\prime} w^{\prime 2}+\frac{1}{3} \rho^{\prime \prime} u v^{\prime \prime 2}+\rho^{\prime} u^{\prime 2}+\rho^{\prime \prime} u^{\prime 2}, \quad . \quad .
$$

which accordingly exceeds the transverse stress by

$$
\kappa=\rho^{\prime} u^{\prime 2}+\rho^{\prime \prime} u^{\prime \prime 2} \text {. . . . . . . . . }
$$

This, therefore, would be the Crookes's stress in the case supposed. It is a very large quantity, since $u^{\prime}$ and $u^{\prime \prime}$ would be large if the streams could penetrate one another without obstruction. The flow of heat, which we will designate by the symbol G, would also be very large in the case supposed. An expression for it can be easily found, but is not required for our present purpose.

11. The other limit is one that really occurs. It arises when the molecules coming up to either piston and those retiring from it form complementary parts, such that their coexistence in the same space constitutes stationary unpolarized gas. This happens only when the two pistons are at the same temperature. In this case it is manifest that no heat is conveyed by the gas, and that the gas exerts the same pressure in all directions. In symbolical language,

$$
\begin{aligned}
& \mathrm{G}=0, \\
& \kappa=0,
\end{aligned}
$$

$G$ being, as before, the symbol for the flow of heat, and $\kappa$ for the Crookes's stress. This case may be described as one in which the streams described in the last section experience such effectual opposition from each other that the speed with which they advance is zero. For it is evident that the gas at any station within the tube may, without any change of its properties, be described as consisting of two equal portions of stationary unpolarized gas coexisting in the same space.

12. In all other cases the pistons that close the ends of the unit tube are at different temperatures, and the gas between any two cross sections of the tube is polarized. Let us consider a slice between two such sections, which are sufficiently close to entitle us to regard the included gas as being throughout in nearly the same state. The actual condition of the gas

* The term "stress" is here applied to the pressure within the gas in any direction, viewed in conjunction with the equal pressure in the opposite direction. It is what Clausius bas called "the positive momentum," meaning thereby the sum of the components of the momenta of the molecules resolved in a given direction, and all estimated as positive, whether of molecules that move forward or backward. 
within the slice may evidently be conceived of as arising from the coexistence of two streams travelling in opposite directions along the tube, and each consisting of gas which is less polarized (i.e. which deviates less from the condition of ordinary gas) than the gas that results from their coexistence. Fach stream is exposed within the slice to the mutual jostling of its own molecules; and it is also attacked by molecules of the other stream. The mutual jostling of its own molecules tends, as explained in section 10 , both to maintain the onward velocity of the stream and to reduce the gas of which the stream consists still more towards the condition of unpolarized gas: These encounters then', taken by themselves, tend to bring about the state of the gas described in section 10. But the interference of the two streams with one another counteracts this. This interference modifies the effect of the encounters within the streams, but it is incompetent to annul it; for the two streams do not by their mere coexistence constitute stationary unpolarized gas, and hence they would need time before they could by their action upon one another reduce the gas to this condition. It is, however, plain that whatever action they exert is a step towards bringing about this condition; for the gas would become depolarized if the cross sections which bound the slice could be rendered impervious both to energy and molecules, so as to leave the two streams time to act fully on one another. In reality, however, sufficient time is not allowed to them, because the streams pass one another, and the struggle is continually renewed within the slice by fresh portions of the streams which come up in the same state as those that had been obliged to pass on. These fresh portions keep in the same state because a sufficient supply of swift molecules is without intermission being thrown back along the tube from one end by the heater, and a corresponding supply of slow molecules from the other end by the cooler.

13. The two streams, however, though not annulled, are different from what they would have been if they had been without influence upon one another. They do not consist of the same molecules from one instant to another; for there is such a perpetual shifting of molecules between them, owing to the vast number of encounters that take place, that no one molecule is likely to remain long in one stream. Again, after an encounter between molecules of the two streams, both of the colliding molecules will sometimes join the same stream; and it will most frequently happen that the stream so joined is the hotter and swifter stream. Hence the stream from the beater to the cooler receires an accession to the number of its 


\section{Mr. G. J. Stoney on Polarization Stress in Gases.}

molecules as it travels forward, while the reverse effect is produced upon the stream making its way in the opposite direction. On both accounts there will be gradients of density and temperature along the tube between the heater and cooler. Again, every encounter between molecules of the two streams diminishes the momentum of one or both streams; but, as we have seen, the effect so produced does not go the length of reducing the streams to rest.

14. Hence we must bear in mind the gradients of temperature and density along the two streams, and the continual fluctuation of the molecules that are to be referred to them, if we want to. regard the condition of the gas throughout the whole length of the tube as arising out of the coexistence of two streams of gas less polarized than itself. But with these precautions the hypothesis may be made; and accordingly the condition of the gas at every cross section of the tube is intermediate between a structure represented by the coexistence of the two streams of unpolarized gas travelling simultaneously in opposite directions, which the encounters within each stream tend to develop, and the condition of stationary unpolarized gas, towards which the mutual interference of the two streams modifies the structure. Hence there is some polarization stress and some flow of heat all along the tube, though of less amount than in the case considered in section 10 . We may still employ equation (7) as the expres:ion for the polarization stress, if we use for $\rho^{\prime}$ and $\rho^{\prime \prime}$ the densities of the streams at some particular cross section of the tube, and if $u^{\prime}$ and $u^{\prime \prime}$ are modified into what they become as the interference of the two streams with one another is increased. It is not necessary to ascertain what this modification will be : it is enough for our purpose to know that $u^{\prime}$ and $u^{\prime \prime}$ will be some functions of $V^{\prime}-V^{\prime \prime}$ (where $V^{\prime 3}$ and $V^{\prime / 3}$ are the averages of the cubes of the velocities of the molecules that pass forwards and backwards respectively through the cross section), and that they will be proportional to this quantity when all three are small.

15. We may base upon this circumstance an investigation into the laws of the phenomenon when the difference between the temperatures of the heater and cooler is small compared with their absolute temperatures. This case is of importance because it is that which most frequently occurs, and is the only one in reference to which accurate experiments have been made. In this case $\rho^{\prime}$ and $\rho^{\prime \prime}$ will each be nearly $\frac{1}{2} \rho$, using $\rho$ for the density of the gas at the position in the tube which we are considering ; and $V^{\prime}-V^{\prime \prime}$, being small, may be appropriately represented by $\delta \mathrm{V}$. Then, remembering that $u^{\prime}$ and $u^{\prime \prime}$ are proportional to $\delta \mathrm{V}$, we obtain from equation (7) 
the following expression for the polarization stress,

$$
\kappa \propto \rho(\delta \mathrm{V})^{2} \text {, }
$$

where the symbol $\propto$ means approximately varies as. Moreover it can be shown* from various considerations that the flow of heat

or, by a simple transformation,

$$
\mathrm{G} \propto \rho \mathrm{V}^{2} \delta \mathrm{V}
$$

$$
\mathrm{G} \propto \rho \mathrm{T} . \delta \mathrm{V}, \quad . \quad . \quad . \quad . \quad .
$$

$\mathrm{T}$ being the temperature measured from absolute zero. Hence from the approximate equations (8) and (9) we obtain the equation

$$
\kappa \propto \frac{\mathrm{G}^{2}}{\rho \mathrm{T}^{2}}, \text {. . . . . . . . . . }
$$

which contains only quantities of which we already know enough to make use of them. Equation (A) may be thrown into a still more convenient form by writing $\mathrm{P}$ for the tension of the surrounding atmosphere of gas, which is nearly the same as the stress which the gas at the station we are consi-

* One of the ways in which this may be proved is the following:Clausius has shown (Phil. Mag. xxiii. p. 514) that

whence

$$
\mathrm{G}=\frac{1}{4} \beta \rho \int_{-1}^{+1} \mathrm{I} \overline{V^{3}} \mu d \mu
$$

$$
G=\frac{1}{8} \beta \rho\left(I^{\prime} V^{\prime 3}-I^{\prime \prime} V^{\prime \prime 3}\right),
$$

where $I^{\prime}$ and $V^{13}$ are the average values of $I$ and $\overline{V^{3}}$ under the integral for positive values of $\mu$ (i.e. for molecules traversing the section of the tube towards the cooler), and $I^{\prime \prime}$ and $V^{\prime \prime 3}$ are the corresponding averages for negative values of $\mu, i . e$. for molecules traversing the section of the tube in the opposite direction.

Now it is evident that these quantities are capable of expansion in the following form :-

$$
\begin{aligned}
& \mathrm{I}^{\prime}=1+\mathrm{A}_{1} \frac{\delta \mathrm{V}}{\mathrm{V}}+\mathrm{A}_{2} \frac{(\delta \mathrm{V})^{2}}{\mathrm{~V}^{2}}+\ldots, \\
& \mathrm{I}^{\prime \prime}=1+\mathrm{B}_{1} \frac{\delta \mathrm{V}}{\mathrm{V}}+\ldots, \\
& \mathrm{V}^{\prime 3}=\mathrm{V}^{3}\left(1+\mathrm{C}_{1} \frac{\delta \mathrm{V}}{\mathrm{V}}+\ldots\right), \\
& \mathrm{V}^{\prime \prime 3}=\mathrm{V}^{3}\left(1+\mathrm{D}_{1} \frac{\delta \mathrm{V}}{\mathrm{V}}+\ldots\right),
\end{aligned}
$$

in which $\mathrm{V}^{9}$ is the average of the values of $\overline{\mathrm{V}^{3}}$ for all directions. Whence

$$
G=\frac{1}{8} \beta p\left(A_{1}-B_{1}+C_{1}-D_{1}\right) V^{2} \delta V
$$

+ terms containing higher powers of $\delta \mathrm{V}$. 
414 Mr. G. J. Stoney on Polarization Stress in Gases.

dering would exert if depolarized. $\mathrm{P}$ will therefore vary nearly as $\rho \mathrm{T}$, whence

$$
\kappa \propto \frac{\mathrm{G}^{2}}{\mathrm{P} \tilde{\mathrm{T}}^{*}} \cdot \cdot \cdot \cdot . \quad . \quad .
$$

16. As an example of the application of these approximate

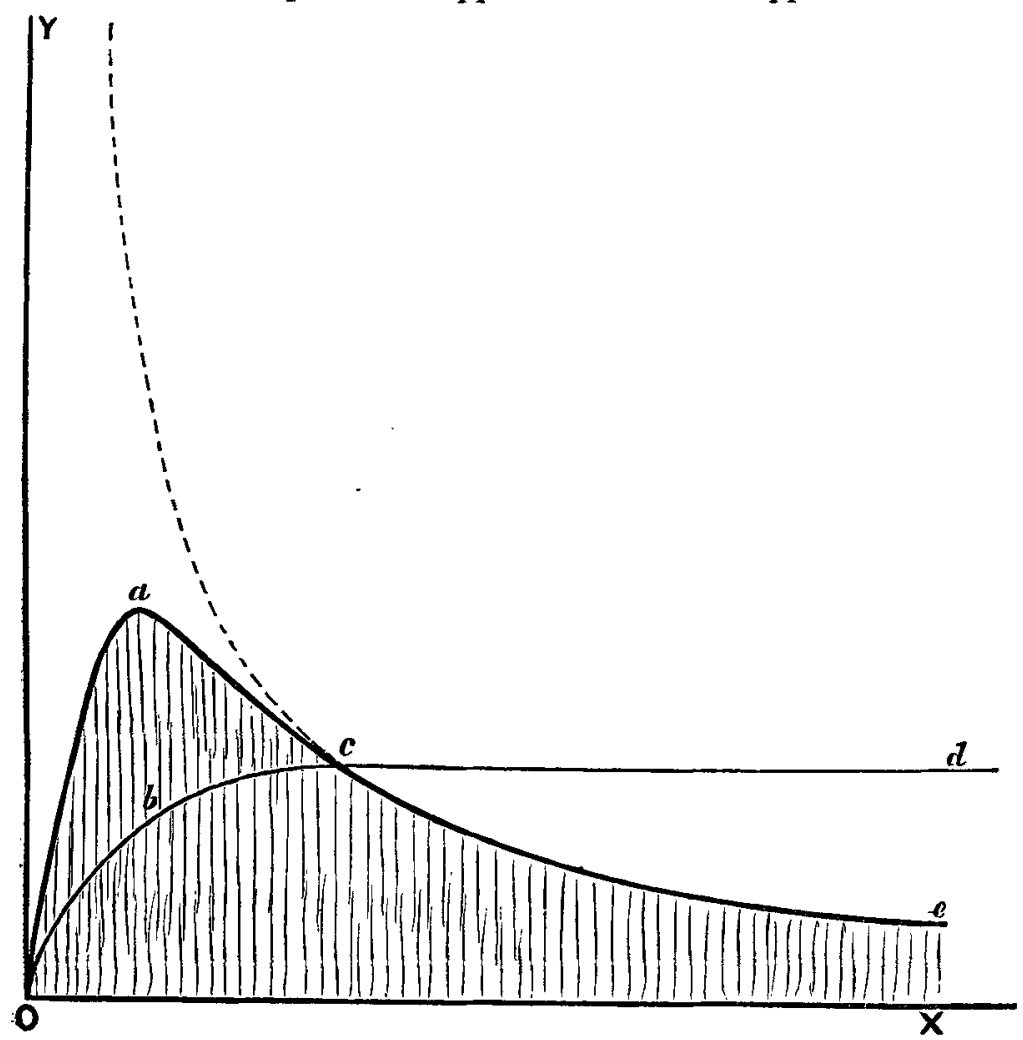

formulæ, let us plot down on a diagram the value of $\kappa$, the polarization stress, for various tensions of gas between a heater and cooler at constant temperatures and at a fixed distance asunder.

Let the abscissas of the figure represent the tensions of the gas. Then the curve $\mathrm{O} b c d$, the ordinates of which represent the flow of heat, is known. The part representing conduction is the parallel line $c d$; and $O b c$ represents the outflow of heat by that modified conduction which may be called penetration, which occurs when the exhaustion has proceeded so far that the number of molecules in a unit tube is less than $N$ (see 
above, section 8). The curve $\mathrm{O} b c d$ is therefore known; and if by equation (B) we plot down from it the values of $\kappa$ (the polarization stress), we find them approximately represented by the ordinates of a curre of the form $\mathrm{O} a c e$, the portion to the right of $c$ being coincident with an equilateral hyperbola, while to the left of $c$ the ordinates fall short of the hyperbola, rising to a maximum and then falling off to zero. The position of this maximum cannot be obtained with certainty, because equation (B) is less to be depended on at very low tensions. Bearing this in mind, the accordance of the theoretic values with those determined experimentally by Mr. Crookes and Mr. Moss is satisfactory.

17. From equation (B) we may obtain another useful formula which expresses the approximate law according to which polarization stress depends upon the interval between heater and cooler, whenever this interval exceeds the limit determined by the condition that there shall be a sufficient number of molecules in the unit tube to allow heat to pass by conduction.

In this case we know the equation of the gradient of temperature (see Clausius' equation (54), Phil. Mag. vol. xxiii. p. 527), and that it is approximately represented by a straight line when, as we have assumed, $\frac{\Delta T}{T}$ is small, using $\Delta \mathrm{T}$ for the difference between the temperatures of the heater and cooler. Hence, and from Clausius' equation (56), it appears that

$$
\frac{\mathrm{G}^{2}}{\mathrm{~T}} \propto \frac{(\Delta \mathrm{T})^{2}}{\mathrm{X}^{2}}
$$

using $\mathrm{X}$ for the distance between the heater and cooler. Introducing this value into equation $(B)$, we find

$$
\kappa \propto \frac{(\Delta \mathrm{T})^{2}}{\mathrm{PX}^{2}}, \text {. . . . . . . }
$$

a result which agrees satisfactorily with Mr. Moss's experiments.

18. If we use $X_{0}$ for that interval between heater and cooler which would make the number of molecules in the unit tube equal to $N$, and if we use $\kappa_{0}$ for the corresponding value of the Crookes's stress, then equation (C), and the obvious equation $\mathrm{X}_{0} \propto \frac{1}{\mathrm{P}}$, furnish us with the following:-

$$
\kappa_{0} \propto \mathrm{P},(\Delta \mathrm{T})^{2} .
$$

Now equation $(C)$ enables us to plot down a part of the curve representing the relation between $\kappa$ and $\mathrm{X}$ when $\Delta \mathrm{T}$ and $\mathrm{P}$ are kept constant; and although equation (C) cannot be relied upon when $X$ is less than $X_{0}$, it is nevertheless evident that 


\section{Mr. G. J. Stoney on Polarization Stress in Gases.}

the shape of the remainder of the curve must be one which is independent of the particular value of $P$ which we have used. Hence, if $\bar{\kappa}$ is the maximum value of $\kappa$ in that curve, it follows that $\bar{\kappa}$ and $\kappa_{0}$ must remain proportional to one another when $P$ is changed. Hence equation (10) furnishes

$$
\bar{\kappa} \propto \mathrm{P} \cdot(\Delta \mathrm{T})^{2} \text {. }
$$

We learn from this inquiry that the maximum polarization stress which can be elicited between a given heater and cooler by varying the distance between them will, if the tension of the gas is altered, change in the same ratio as that tension, and that it will occur at intervals between heater and cooler which vary inversely as that tension. This fully accounts for the powerful Crookes's force which presents itself in experiments at ordinary atmospheric tensions as compared with the feeble force exhibited in radiometers. It accounts also for the very short interval at which the heater and cooler must be placed when the gas is dense.

Part II. Investigation of a Complete Expression for the Stress.

19. As it has been asserted ('Nature,' vol. xvii. p. 122) that the views of the present writer are at variance with the results established by previous investigators, I will proceed to show that the theory of unequal stresses which I have put forward is, on the contrary, the necessary sequel of them. I will show this by continuing the method of investigation commenced by Professor Clausius in his memoir on " the Conduction of Heat by Gases," in the way which was pointed out by Mr. George F. Fitzgerald in 'Nature,' vol. xvii.p. 200. This inquiry will have the further advantage of furnishing a complete expression for Crookes's stress.

Clansius (Phil. Mag. vol. xxiii. p. 514) has given the following expression for the stress across a layer of gas conducting heat, in the direction normal to a heater and cooler, the opposed surfaces of which are parallel and extensive,

$$
\mathrm{P}_{x}=\frac{1}{3} \rho v_{y}^{2}+\mathrm{X}_{1} \epsilon^{2}
$$

$\boldsymbol{\epsilon}$ being a small quantity of the same order as the strikingdistance of the molecules, and $X_{1}$ being a coefficient of which Clausius did not compute the value, as the scope of his investigation only required him to go as far as the first order of small quantities.: Now Mr. Fitzgerald, in his letter to ' Nature,' and more fully in conversation with the writer, pointed out that if an expression for $\mathrm{P}_{y}$, the stress parallel to the surfaces of the heater and cooler, were calculated by a method similar to Clausius', the coefficient of $e^{2}$ in this expression could not 
be the same as $X_{1}$, and that hence there must be a difference between the two stresses-in other words, a polarization stress. 20. Clausius (loc. cit.) gives the following general expression for the normal stress-

$$
\mathrm{P}_{x}=\frac{1}{2} \rho \int_{-1}^{+1} 1 \overline{\mathrm{V}^{2}} \mu^{2} d \mu, \text {. . . . . }
$$

where $I$ is the coefficient expressing the proportion of molecules travelling in the directions which make an angle with the normal or axis of $x$ of which the cosine is $\mu$, and where $\overline{\mathrm{V}}^{\mathrm{e}}$ is the mean of the squares of their velocities.

Now if, employing a process exactly similar to that pursued by Clausius on pp. 512 and 513 of his memoir, we use $N$ for the number of molecules in a unit of volume, then will $\mathrm{N} d r$ be the number of molecules within a slice of unit area and thickness $d r$, which we may suppose to be placed perpendicular to the vector $r$. Then

$$
\frac{1}{4 \pi} \mathrm{NI} d r d \sigma
$$

will be the number of molecules moving within the slice in directions which lie within an element of solid angle $d \sigma$, which we will suppose makes the angle $\psi$ with the vector $r$; so that the time they take to cross the slice will be

$$
\frac{d r \cdot \sec \psi}{\mathrm{V}}
$$

$\mathrm{V}$ being their velocity. Hence the number traversing the slice in the specified direction within a unit of time is

$$
\frac{1}{4 \pi} \cdot \text { NIV } \cos \psi \cdot d \sigma \text {. }
$$

Multiplying this by $m \mathrm{~V} \cos \psi$ we get the resolved part of their momenta along $r$. The sum of all such components of the momenta, all estimated as positive, is $P_{r}$, the stress in the direction of $r$. Whence, and writing $\rho$ for $m \mathrm{~N}$, we find

$$
\mathrm{P}_{x}=\frac{\rho}{4 \pi} \iint \mathrm{I} \overline{\mathrm{V}}^{2} \cos ^{2} \psi d \sigma,
$$

the integration being extended over the unit sphere.

Hence the stresses in the directions of three rectangular axes are

$$
\begin{aligned}
& \mathrm{P}_{x}=\frac{\rho}{4 \pi} \iint I \overline{V^{2}} \cos ^{2} \alpha d \sigma, \\
& \mathrm{P}_{y}=\frac{\rho}{4 \pi} \iint I \overline{V^{2}} \cos ^{2} \beta d \sigma, \\
& \mathrm{P}_{x}=\frac{\rho}{4 \pi} \iint I \overline{V^{2}} \cos ^{2} \gamma d \sigma,
\end{aligned}
$$

Phil. Mag. S. 5. Vol. 6. No. 39. Det. 1878. 
$\alpha, \beta, \gamma$ being the director angles of the element of solid angle $d \sigma$. Introducing polar coordinates, we have

$$
\begin{aligned}
d \sigma & =\sin \theta d \theta d \phi, \\
\cos \alpha & =\cos \theta, \\
\cos \beta & =\sin \theta \cos \phi, \\
\cos \gamma & =\sin \theta \sin \phi,
\end{aligned}
$$

by which the expressions for the stresses become

$$
\left.\begin{array}{l}
\mathrm{P}_{x}=\frac{\rho}{4 \pi} \int_{0}^{2 \pi} \int_{0}^{\pi} I \overline{V^{2}} \cos ^{2} \theta \sin \theta d \theta d \phi, \\
\mathrm{P}_{y}=\frac{\rho}{4 \pi} \int_{0}^{2 \pi} \int_{0}^{\pi} I \overline{V^{2}} \sin ^{3} \theta \cos ^{2} \phi d \theta d \phi, \\
\mathrm{P}_{z}=\frac{\rho}{4 \pi} \int_{0}^{2 \pi} \int_{0}^{\pi} I \overline{V^{2}} \sin ^{3} \theta \sin ^{2} \phi d \theta d \phi .
\end{array}\right\} .
$$

These are the most general expressions for the stresses in three rectangular directions within gas polarized in any way; and they will be the only stresses between portions of the gas separated by planes parallel to the planes $y z, z x, x y$, if the axes are so chosen that there are no moments round them arising from the molecular encounters*.

21. 'This condition is easily secured in the case which we are investigating, viz. when heat is making its way between a heater and cooler that are parallel to one another, and of large extent compared with the interval between them, since the polarization of the intervening gas will evidently be disposed symmetrically round the direction in which the heat is travelling. Hence, taking this direction as our axis of $x$, there can be no moments round this axis, or round any axis at right angles to it. The stresses $(\mathrm{E})$, therefore, are the only ones to be taken into account. Moreover we can integrate equations (E) at once by $\phi$, since $I \bar{V}^{2}$ is, in this simple case, a function

* Equations ( $(\mathrm{E})$ cannot be integrated unless $I \overline{V^{2}}$ is given as a function of $\theta$ and $\phi$, i. e. mless the law of polarization in the gas is known. But they show that in general the stresses in different directions are unequal, which is here what is chiefly insisted on.

When the gas is unpolarized, I becomes equal to unity, and $\overline{\mathrm{V}^{2}}$ is independent of the direction, and may therefore be put outside the integrals. In this case all three equations concur in giving the well-known expression for the stress in uppolarized gas, viz. $\frac{1}{3} \rho \overline{V^{2}}$. 
Mr. G. J. Stoney on Polarization Stress in Crases.

of $\theta$ only. Doing this, and writing $\mu$ for $\cos \theta$, we find

$$
\left.\begin{array}{l}
\mathrm{P}_{x}=\frac{\rho}{2} \cdot \int_{-1}^{+1} \mathrm{I} \overline{\mathrm{V}^{2}} \mu^{2} d \mu, \\
\mathrm{P}_{y}=\mathrm{P}_{z}=\frac{\rho}{4} \int_{-1}^{+1} I \overline{V^{2}}\left(1-\mu^{2}\right) d \mu,
\end{array}\right\}
$$

whence, since $\kappa$, the polarization stress, $=\mathrm{P}_{x}-\mathrm{P}_{y}$, we have finally

$$
\kappa=\frac{\rho}{4} \int_{-1}^{+1} I \overline{V^{2}}\left(3 \mu^{2}-1\right) d \mu . \quad . \quad . \quad .
$$

This, then, is the complete mathematical expression for Crookes's stress. It could be integrated if we knew the law of the polarization of the gas; for then $I \overline{V^{2}}$ would be a known function of $\mu$.

22. Clausius, in investigating the diffusion of heat across the layer of gas, makes the assumption (Phil. Mag. vol. xxiii. pp. 425 and 524 ) that the numbers and velocities of the molecules" emitted". by a thin stratum of the gas (i.e. that have passed out of the stratum after having encountered other molecules within it) may be adequately represented "by assuming at first motions taking place equally in all directions, and then supposing a small additional component velocity in the direction of positive $x$ to be imparted to all the molecules." In other words, it is assumed that the motions of these molecules may be represented by radii vectores from a slightly excentric origin to points equally distributed over the surface of a sphere. It will be instructive to trace the consequences of this hypothesis, both because of what it will do and what it will not do.

Upon this hypothesis Clausius finds the following convergent series for $\overline{V^{2}}$ and I (loc. cit. pp. 434 and 516):-

$$
\begin{aligned}
& \overline{\mathrm{V}^{2}}=u^{2}+2 u q \mu \epsilon+\left(2 u r+q_{1}^{2}\right) \mu^{2} \epsilon^{2}+\ldots, \\
& \mathrm{I}=\left(1-\frac{1}{3} r^{\prime} \epsilon^{2}+\ldots\right)-\frac{q}{u} \cdot \mu \epsilon+r^{\prime} \mu^{2} \varepsilon^{4}+\ldots,
\end{aligned}
$$

where $\frac{1}{5} q \epsilon$ (loc. cit. p. 525) is the small component velocity spoken of above, $u$ is the mean velocity of molecules moving in the plane $y z$, and the other letters have the meanings assigned to them by Clausius. Multiplying these together, going to the second order of small quantities, and arranging by powers of $\mu$, we find

where

$$
I \overline{V^{2}}=u^{2}\left(1-\frac{1}{s} r^{\prime} \epsilon^{2}\right)+A_{1} \mu \epsilon+A_{2} \mu^{2} \epsilon^{2}, \quad .
$$

$$
\begin{gathered}
\mathrm{A}_{2}=-2 q^{2}+2 u r^{\prime}+q_{1}^{2}+u^{2} r^{\prime} . \\
2 \mathrm{E} 2
\end{gathered}
$$


420 Mr. G. J. Stoney on Polarization Stress in Gases.

Introducing the expression (12) into equations (F) and $(G)$ we find

$$
\left.\begin{array}{rl}
\mathrm{P}_{x} & =\frac{1}{3} \rho u^{2}\left(1-\frac{1}{3} r^{\prime} \epsilon^{2}\right)+\frac{1}{5} \rho \mathrm{A}_{2} \mathrm{e}^{2}+\ldots, \\
\mathrm{P}_{y} & =\mathrm{P}_{z}=\frac{1}{3} \rho u^{2}\left(1-\frac{1}{3} r^{\prime} \epsilon^{2}\right)+\frac{1}{15} \rho \mathrm{A}_{2} \epsilon^{2}+\ldots, \\
\kappa & =\frac{2}{15} \rho A_{2} \epsilon^{2}+\ldots .
\end{array}\right\} .
$$

In these $\mathrm{A}_{2}$ stands for the expression (1.3); and introducing the following values, which are given by Clausius as correct to the second order of small quantities (loc. cit. p. 526, footnote),

$$
\begin{aligned}
q_{1}^{2} & =\frac{41}{25} q^{2}, \\
u r & =-\frac{31}{50} q^{z}, \\
u^{2} r^{\prime} & =\frac{266}{17 \cdot 25} q^{2},
\end{aligned}
$$

we find

From this and (14),

$$
\mathrm{A}_{2}=13 \cdot 8 q^{2} \text {. }
$$

$\kappa=1.8 \times \rho q^{2} \epsilon^{2}+$ terms of the fourth and higher orders.

But by Clausius' theory (loc. cit.p. 516),

$\mathrm{G}=\frac{1}{3} \beta \rho u^{2} q \epsilon+$ terms of the third and higher orders. . (16)

Whence, approximately, omitting the fourth and higher orders of small quantities, and writing $v$ for $u$, since they are nearly equal, and then putting $P$ for its equivalent $\frac{1}{3} \rho v^{2}$,

$$
\kappa=1 \cdot 8 \frac{\rho \mathrm{G}^{2}}{\beta^{2} \mathrm{P}^{2}} \cdot \text {. . . . . . }
$$

Now, by Boyle and Charles's law,

$$
\frac{\mathrm{P}}{\mathrm{P}_{0}}=\frac{\rho \mathrm{T}}{\rho_{0} \mathrm{~T}_{0}}
$$

where $P_{0}, \rho_{0}$, and $T_{0}$ have reference to standard temperature and pressure. Whence, finally,

$$
\kappa=\left[(1 \cdot 8) \frac{\rho_{0} \mathrm{~T}_{0}}{\beta^{2} \mathrm{P}_{0}}\right] \cdot \frac{\mathrm{G}^{2}}{\mathrm{PT}}, \cdot \ldots .
$$

an equation which assigns the same law as we obtained above in equation (B) by the wholly different method of direct mechanical considerations.

23. Equation (18) appears to give also the amount of the polarization stress. But this is illusory. The hypothesis upon which it rests is adequate as regards the conduction of heat, but is insufficient for a quantitative investigation of the stress, as I will now proceed to show. 
The general formulæ for the conduction of heat and for the polarization stress are the following-

$$
\begin{aligned}
& \mathrm{G}=\frac{1}{4} \beta \rho \int_{-1}^{+1} \mathrm{I} \overline{V^{3}} \cdot \mu d \mu, \\
& \kappa=\frac{1}{4} \rho \int_{-1}^{+1} \mathrm{I} \overline{V^{2}} \cdot\left(3 \mu^{2}-1\right) d \mu
\end{aligned}
$$

(see Clausius' memoir, p. 514, and equation (G) above). Now $\mu$ and $3 \mu_{2}-1$, which occur as factors in these integrals, are the first and second terms of a series of spherical harmonics (Laplace's coefficients) of the simple kind that are functions of $\mu$ only, and which therefore represent the radii of solids of revolution from points on their axes. It is moreover obvious that we can expand $I \bar{V}^{2}$ and $I \overline{V^{3}}$ in series of spherical harmonics of the same simple type. Doing this,

$$
\begin{aligned}
& \mathrm{I} \overline{V^{3}}=g_{0}+g_{1}+g_{2}+\ldots, \\
& \mathrm{I} \bar{V}^{\overline{2}}=k_{0}+k_{1}+k_{2}+\ldots,
\end{aligned}
$$

the $g$ 's and $k$ 's representing spherical harmonics. Whence, and from the fundamental property of spherical harmonics,

$$
\begin{aligned}
& \mathrm{G}=\frac{1}{4} \beta \rho \int_{-1}^{+1} g_{1} \mu \delta \mu, \\
& \kappa=\frac{1}{4} \rho \int_{-1}^{+1} k_{2}\left(3 \mu^{2}-1\right) d \mu .
\end{aligned}
$$

Hence $g_{1}$ is the only term of the first series that produces any conduction of heat, and $k_{2}$ is the only term of the second series that produces any polarization stress.

Let us suppose radii drawn from a point in all directions, of lengths proportional to the values of $\mathrm{IV}^{2}$ in those directions. We thus obtain a solid of revolution which may also be arrived at by plotting down radii equal to $k_{0}$, and successively correcting the solid so found by the addition of $k_{1}, k_{2}$, \&c. to its radii. Now

$$
\begin{aligned}
& k_{0}=\mathrm{A}, \\
& k_{1}=\mathrm{B} \cdot \mu, \\
& k_{2}=\mathrm{C} \cdot\left(3 \mu^{2}-1\right), \\
& \text { \&c. } \quad \text { \&c. }
\end{aligned}
$$

where $A, B, C, \& c$. are independent of $\mu$. In the case we are considering, B, C, \&c. are small compared with A. From the foregoing values it follows that if $k_{0}$ is plotted down by itself, it will produce a sphere with its centre at the origin of radii. 
Next, $k_{0}+k_{1}$ may be plotted down by shifting the centre of this sphere through the small distance $\mathrm{B}$ towards positive $x$, and by then very slightly distorting the form of the sphere. Again, to plot down $k_{0}+k_{2}$, we should elongate the sphere in the direction of the axis $x$ by an amount equal to $4 \mathrm{C}$, and narrow it equatorially by an amount equal to $2 \mathrm{C}$, without shifting its centre. Finally, $k_{0}+k_{1}+k_{2}$ would be represented by radii drawn to the surface of this last solid, after it had been slightly distorted and removed through the distance $B$ towards the cooler. Through all these mutations the mean value of the radii drawn from the origin remains unaltered*.

Comparing these figures with expansion (12), which is the value for $I \bar{V}^{2}$ furnished by Clausius' hypothesis, we find that the form and position of the solid which results from plotting it down are such that (owing to the term containing $\mu$ ) there is that separation between the origin of radii and the centre of figure which gives a sufficient value to the function $k_{1}$, but that (the coefficient of $\mu^{2}$ containing only very small quantities) the solid is not elongated in the way which would allow the function $k_{2}$ to attain any considerable value. That the function $k_{2}$ is not wholly absent is because of such causes as the slight distortion of figure before mentioned, which give rise to very small $\dagger$ terms of the form $k_{1}, k_{2}$, \&c.

* Since, by the fundamental property of spherical harmonics,

$$
\begin{aligned}
& \int_{-1}^{+1} k_{1} d \mu=0, \\
& \int_{-1}^{+1} k_{2} d \mu=0,
\end{aligned}
$$

$\&$ c.

† That $k_{2}$ is very small, if we adopt Clausius' hypothesis, may also be seen by comparing equation (18) with experiments on spheroidal drops. Observation shows that, at atmospheric temperatures and pressures, a spheroid of water some millimetres in diameter will be supported at a distance of about a fourth-metre (a metre divided by 104) from the heater, when the difference of temperatures is about $10^{\circ} \mathrm{C}$. In G.C.S. (gramme, centimetre, second) systematic measures, the hyper-milligram $\left(\frac{10}{g}\right.$ of the gravitation of a milligram, $g$ being gravity measured in metres per second) per square centimetre is the unit of stress. Hence the Crookes's stress which supports this drop must amount to some hundreds of these units. This is the amount indicated by experiment.

Formula (18) assigns to it a very different value. Clausius estimates the flow of heat across air between a heater and cooler, each a square metre in surface and a metre asunder, and kept at temperatures which differ by $1^{\circ} \mathrm{C}$, as amounting per second to $\frac{11}{400000}$ of the quantity of heat which will warm a kilogram of water $1^{4} 0^{\circ}$. A A bout ten times this, or $\frac{11}{400000}$ of this calory per second, would be the flow of heat between 
This almost total absence of the elongated form arises from Clausius' fundamental hypothesis, that the motions of the molecules emitted by a stratum may be represented by radii drawn to a sphere from an excentric point; whereas it appears from the discussion in the earlier part of the present memoir that the encounters that take place within each of the two streams into which the gas may be divided, give to the surface to which the radii are to be drawn an elongated form. This omission from Clausius' hypothesis does not sensibly affect the spherical harmonics of the first order, and accordingly his hypothesis is adequate as regards the flow of heat, which depends exclusively on one of these ; but it renders the hypothesis an insufficient one as regards polarization stress, or any other phenomenon which depends on spherical harmonics of the second order.

LIV. Researches on Unipolar Induction, Atmospheric Electricity, and the Aurora Borealis. By E. EDLund, Professor of Physics at the Swedish Royal Academy of Sciences.

[Concluded from p. 371.]

A $\mathrm{S}$ the magnetic properties of the earth cannot be fully accounted for by assuming the existence of a magnet in its interior, it is quite evident that the preceding consideration can only indicate the general course of the phenomena in question. We will now rapidly examine whether the results of that consideration are conformable to those given by observation.

Atmospheric electricity has long been the object of repeated investigations. These have been so numerous that to give an

two square centimetres at the distance of a fourth-metre asunder and kept at temperatures that differ by $10^{\circ} \mathrm{C}$. To turn this into kinetic measure we must multiply by $41600 \times 1000000$; so that $\mathrm{G}$ would amount to about 1144000 in G.O.S. kinetic measure ( $i$. $e$. in hyper-fifth-grammetres per. second). Again, we may take as rough approximations,

$$
\begin{aligned}
& \rho_{0}=\frac{1}{800}, \\
& \mathrm{~T}_{0}=\mathrm{T} . \\
& \beta^{2}=2 \cdot 6, \\
& \mathrm{P}_{0}=\mathrm{P}_{1}=1000000 .
\end{aligned}
$$

Introducing these values into equation (18), we find approximately,

$$
k=0.001
$$

of a hyper-milligram per square centimetre-an amount which, as it ought to be, is vastly smaller than that indicated by experiment. 